\title{
Inference for Long-memory Time Series Models Based on Modified Empirical Likelihood
}

\author{
Ramadha D. Piyadi Gamage \\ Western Washington University
}

\author{
Wei Ning \\ Bowling Green State University
}

\begin{abstract}
Empirical likelihood method has been applied to short-memory time series models by Monti (1997) through the Whittle's estimation method. Yau (2012) extended this idea to long-memory time series models. Asymptotic distributions of the empirical likelihood ratio statistic for short and long-memory time series have been derived to construct confidence regions for the corresponding model parameters. However, it experiences the undercoverage issue which causes the coverage probabilities of parameters lower than the given nominal levels, especially for small sample sizes. In this paper, we propose a modified empirical likelihood which combines the advantages of the adjusted empirical likelihood and the transformed empirical likelihood to modify the one proposed by Yau (2012) for autoregressive fractionally integrated moving average (ARFIMA) model for the purpose of improving coverage probabilities. Asymptotic null distribution of the test statistic has been established as the standard chi-square distribution with the degree of freedom one. Simulations have been conducted to investigate the performance of the proposed method as well as the comparisons of other existing methods to illustrate that the proposed method can provide better coverage probabilities especially for small sample sizes.
\end{abstract}

Keywords: modified empirical likelihood, long-memory time series, ARFIMA model, coverage probability .

\section{Introduction}

Owen (1988), Owen (1990), Owen (1991) introduced empirical likelihood (EL) method which is the data-driven method combining the advantages of parametric and nonparametric methods. The most appealing property of the EL method is that the associated empirical likelihood ratio statistics asymptotically follows standard chi-square distribution, which is same as the one used in parametric analysis. Since then, it has been widely used to make statistical inference of parameters and construct confidence regions. See Owen (2001) for more details. However, when the data is dependent, it becomes difficult to apply the empirical likelihood method as it is originally designed for independent observations. Using EL method to address dependent data problems has been studied by many researchers. Mykland (1995) established the connection between the dual likelihood and the empirical likelihood through the martingale estimating equations and applied it to time series model. Monti (1997) developed the idea of extending the EL method to short-memory stationary time series by using the Whittle's 
(1953) method to obtain an M-estimator of the periodogram ordinates of time series models which are asymptotically independent. However, his method can not be applied directly to long-time memory time series model. Kitamura (1997) developed the blockwise empirical likelihood method for time series models. For long-memory or long-range dependence time series data, Hurvich and Beltrao (1993) showed that the normalized periodogram ordinates obtained from a Gaussian process are asymptotically neither independent identically distributed nor exponentially distributed. Nordman and Lahiri (2006) developed frequency domain empirical likelihood based on the spectral distribution through the fourier transformation to study short and long range dependence. Yau (2012) extended Monti's idea to autoregressive fractionally integrated moving average (ARFIMA) model by showing that the dependence in periodogram only applies to a small portion of the periodogram ordinates with fourier frequencies tending to zero. However, the profile empirical likelihood function computation which involves constrained maximization requires the convex hull of the estimating equation to have zero vector as an interior point. When the solution does not exist, Owen (2001) suggested assigning $-\infty$ to the log-EL statistic. Chen, Variyath, and Abraham (2008) pointed out the drawbacks in doing so and proposed an adjusted empirical likelihood (AEL) method by adding a pseudo term which always guarantees the existence of a solution. They further showed that the asymptotic results of the AEL are similar to that of the EL. Moreover, it achieves improved coverage probabilities without using Bartlett-corrections. Adapting their method, Gamage, Ning, and Gupta (2017) proposed an adjusted empirical likelihood procedure to modify the one proposed by Yau for the ARFIMA model and achieved better coverage probabilities of parameters. Jing, Tsao, and Zhou (2017), instead of considering the convex hull issue, developed the transformed empirical likelihood (TEL) to improve the coverage probabilities as well as preserving the properties of the original EL method with a simple form.

In this paper, we extend Yau's EL method for ARFIMA model by proposing a transformed adjusted empirical likelihood method (TAEL). The rest of the article is organized as follows. In Section 2, we briefly go over the AEL procedure proposed by Gamage et al. (2017) for a stationary ARFIMA model. In section 3, we proposed a transformed adjusted empirical likelihood (TAEL) based on the AEL by Chen et al. (2008) and the TEL by Jing et al. (2017) for a stationary ARFIMA model. Corresponding asymptotic results are also discussed. Simulations are carried out in section 4 to compare the coverage probabilities of the proposed TAEL method to other methods including original EL, TEL, and AEL for different distributions for the white noise term. In addition, coverage probabilities are calculated to illustrate the effectiveness of AEL method as compared to EL method with and without Bartlett-correction for different values of the parameters, different sample sizes and different distributions for the white noise term. Section 5 provides some discussion.

\section{Adjusted empirical likelihood for ARFIMA models}

With the definition of $p_{j}=P\left(X=x_{j}\right)$, the empirical likelihood ratio for any value $\beta \in \mathbb{B}$ is defined by

$$
\hat{\lambda}(\beta)=\sup \prod_{j=1}^{n} p_{j} / \prod_{j=1}^{n} \frac{1}{n}=\sup \prod_{j=1}^{n} n p_{j},
$$

subject to the constraints:(i) $\sum_{j=1}^{n} \psi\left(x_{j}, \beta\right) p_{j}=0$, (ii) $\sum_{j=1}^{n} p_{j}=1$, and (iii) $p_{j} \geq 0$, $(j=1,2, \ldots n)$. The maximization under the Lagrange multiplier method gives

$$
p_{j}=\left[n\left\{1+\xi(\beta)^{\prime} \psi\left(x_{j}, \beta\right)\right\}\right]^{-1} \quad(j=1,2, \ldots, n),
$$

where $\xi(\beta)$ is the Lagrangian multiplier satisfying constraint (i). The empirical likelihood ratio statistic is thus defined

$$
\hat{W}(\beta)=-2 \ln \hat{\lambda}(\beta)=2 \sum_{j=1}^{n} \ln \left\{1+\xi(\beta)^{\prime} \psi\left(x_{j}, \beta\right)\right\}
$$


Owen (1988) showed that $\hat{W}(\beta)$ is asymptotically distributed as $\chi^{2}$. For time series data, the M-estimator, $\beta_{0}$, which is used to estimate the parameter $\beta$ is based on the periodogram using the Whittle's method. It is the solution of

$$
\sum_{i=1}^{n} \psi\left(x_{i}, \beta\right)=0
$$

where $\psi\left(x_{i}, \beta\right)$ is an estimating equation which is defined by $E \psi\left(X_{i}, \beta\right)=0$. Under some moment conditions on estimating equations (Owen, 2001), the convex hull of the estimating equation $\psi\left(x_{i}, \beta\right),\left\{\sum p_{i} \psi\left(x_{i}, \beta\right) \mid p_{i} \geq 0, \sum p_{i}=1, i=1,2, \cdots, n\right\}$ contains 0 as its interior point with probability 1 as $n \rightarrow \infty$. When the parameter $\beta$ is not close to the true value $\beta_{0}$, or when $n$ is small, there is a good chance that the solution to constraint doesn't exist which raises some computational issues as mentioned by Chen et al. (2008). When the solution does not exist, Owen (2001) suggested assigning $-\infty$ to the empirical log-likelihood ratio statistic. To overcome this difficulty, Chen et al. (2008) proposed an adjusted empirical likelihood (AEL) ratio function by adding a pseudo term, $\psi_{n+1}$-th term, to guarantee the zero to be an interior point of the convex hull of estimating equations, therefore, the required numerical maximization always has the solution. By doing so, they modified Owen's method and applied to independent observations with the establishment of the Wilks' theorem for AEL statistic same as the one Owen obtained for EL statistic.

The EL-based method proposed by Monti (1997) which used the asymptotically independent periodogram ordinates, cannot be directly used in the long-memory scenario. However, Yau (2012) showed that the periodogram ordinates obtained by the long-memory time series can be considered asymptotically independent, hence the EL method could be extended to the long-memory time series models. Consider a stationary $\operatorname{ARFIMA(p,d,q)~process~} Z_{t}$ given by

$$
\Phi(B)(1-B)^{d} Z_{t}=\Theta(B) a_{t},
$$

for some $-\frac{1}{2}<d<\frac{1}{2}$ where $B$ is the backward shift operator $\left(B Z_{t}=Z_{t-1}\right)$, with $\Theta(B)=$ $1+\theta_{1} B+\theta_{2} B^{2}+\ldots+\theta_{q} B^{q}$ and $\Phi(B)=1-\phi_{1} B-\phi_{2} B^{2}-\ldots-\phi_{p} B^{p}$. The absolute values of the roots of these two polynomials are all greater than 1 to guarantee the stationarity and invertibility of the model. We also assume that $\Theta(B)$ and $\Phi(B)$ have no common factors to avoid the redundancy of the parameters. Similar to Gamage et al. (2017), we consider the values of $d$ to be between 0 and 0.5 since this is the only condition under which the dependence structure of periodogram ordinates has been established (Yau 2012).

Let $\beta=\left(\beta_{(1)}, \sigma^{2}\right)$, where $\beta_{(1)}=\left(\phi_{1}, \ldots, \phi_{p}, \theta_{1}, \ldots, \theta_{q}, d\right)$ is the parameter of interest. Denote $\psi_{j}=\psi_{j}(\beta)=\psi\left(x_{j}, \beta\right)$ and $\bar{\psi}_{n}=\bar{\psi}_{n}(\beta)=\frac{1}{n} \sum_{j=1}^{n} \psi_{j}$. For some positive constant $a_{n}$, define

$$
\psi_{n+1}=\psi_{n+1}(\beta)=-\frac{a_{n}}{n} \sum_{j=1}^{n} \psi_{j}=-a_{n} \bar{\psi}_{n} .
$$

Here we choose $a_{n}=\max (1, \log (n) / 2)$ coupled with a trimmed version of $\bar{\psi}_{n}$ when appropriate suggested by Chen et al. (2008).

Gamage et al. (2017) extended the EL approach for the long-memory time series models and proposed an adjusted empirical likelihood (AEL) ratio statistic as follows.

$$
\hat{W}^{*}\left(\beta_{(1)}\right)=4 \sum_{j=1}^{n+1} \ln \left[1+\xi\left(\beta_{(1)}\right)^{\prime} \tilde{\psi}_{j}\left(I\left(\omega_{j}\right), \beta_{(1)}\right)\right],
$$

where $\tilde{\psi}$-function defined as

$$
\tilde{\psi}_{j}\left\{I\left(\omega_{j}\right), \beta_{(1)}\right\}=\frac{I\left(\omega_{j}\right)}{g_{j}^{1}\left(\beta_{(1)}\right)} \frac{\partial \ln \left\{g_{j}^{1}\left(\beta_{(1)}\right)\right\}}{\partial \beta_{(1)}},
$$


with $\tilde{\psi}_{n+1}$ as defined above, and $\xi\left(\beta_{(1)}\right)$ satisfies,

$$
\sum_{j=1}^{n+1}\left[1+\xi\left(\beta_{(1)}\right)^{\prime} \tilde{\psi}_{j}\left\{I\left(\omega_{j}\right), \beta_{(1)}\right]^{-1} \tilde{\psi}_{j}\left\{I\left(\omega_{j}\right), \beta\right\}=0 .\right.
$$

Here $g_{j}^{1}$ is the profile spectral density function of the model, and $I\left(\omega_{j}\right)$ is the periodogram ordinate evaluated at Fourier frequency $\omega_{j}$. Same discuss as Chen et al. (2008), because $\overline{\tilde{\psi}}_{n}=$ $\frac{1}{n} \sum_{i=1}^{n} \tilde{\psi}_{i}$ and $\tilde{\psi}_{n+1}$ are on the opposite of 0 , therefore, the convex hull of $\tilde{\psi}_{i}, i=1,2, \cdots, n+$ 1 , that is, $\left\{\sum p_{i} \tilde{\psi}_{i} \mid p_{i} \geq 0, \sum p_{i}=1\right\}$, contains 0 as an interior point for any given $\beta_{(1)}$. Consequently, $W^{*}\left(\beta_{(1)}\right)$ is well defined for any given $\beta_{(1)}$. Moreover, $\hat{W}^{*}\left(\beta_{(1)}\right)$, defined in (3) has an asymptotic $\chi^{2}$ with $k=p+q+1$ degrees of freedom (Gamage et al. 2017). As mentioned by Yau (2012), this result also applies to ARFIMA process with $d \in[\delta, 0.5-\delta]$ for any fixed $\delta>0$, since $\beta$ belongs to a compact space.

\section{Transformed adjusted empirical likelihood for ARFIMA models}

The undercoverage problem in the EL method where the coverage probability tends to be lower than the nominal level, is partly due to the convex hull constraint which defines the confidence region inside a bounded region in the parmeter space (Tsao 2013). This could be serious especially in small sample and in multidimensional scenarios. A transformed version of the EL (TEL) has been proposed by Jing et al. (2017) as a solution to the undercoverage problem in EL as follows. Define

$$
g_{T}(l(\theta) ; \gamma)=l(\theta) \times \max \{1-l(\theta) / n, 1-\gamma\},
$$

for a constant $\gamma \in[0,1]$, where $l(\theta)$ is the original empirical log-likelihood ratio (Owen 2001). Thus $g_{T}(l(\theta) ; \gamma)$ is a truncated quadratic transformation of $l(\theta)$. By default, Jing et al. (2017) sets $\gamma=1 / 2$. Hence, the transformed empirical log-likelihood ratio (TEL), $l_{T}(\theta)$ is,

$$
l_{T}(\theta)=g_{T}(l(\theta) ; \gamma=1 / 2)=l(\theta) \times \max \{1-l(\theta) / n, 1 / 2\},
$$

which is equivalent to,

$$
\ell_{T}(\theta)= \begin{cases}\ell(\theta)[1-\ell(\theta) / n] & \ell(\theta) \leq n / 2 \\ \ell(\theta) / 2 & \ell(\theta)>n / 2 .\end{cases}
$$

Jing et al. (2017) showed that the confidence region based on $g_{T}(l(\theta), \gamma=1 / 2)$ is the expansion of the original empirical likelihood confidence region, that is, $\{\theta: l(\theta) \leq c\} \subseteq\{\theta$ : $\left.g_{T}(l(\theta), \gamma=1 / 2) \leq c\right\}$ where $c$ is the $(1-\alpha)$ - th quantile of $\chi_{d}^{2}$. Consequently, TEL improves the coverage probabilities of EL. Moreover, Jing et al. (2017) also showed that TEL shares the same asymptotic properties with the EL. See Jing et al. (2017) for more details.

Consider the ARFIMA (p,d,q) model in (2) where the AEL ratio test statistic is given by (3) which has an asymptotic $\chi^{2}$ distribution with $p+q+1$ degrees of freedom. If we apply the transformation to this test statistic, then the resulting transformed AEL (TAEL) ratio statistic, $W_{T}\left(\beta_{(1)}\right)$, satisfies the following criterions:

a. for any $\beta_{(1)}, 0 \leq W_{T}\left(\beta_{(1)}\right) \leq \hat{W}^{*}\left(\beta_{(1)}\right)$,

b. $W_{T}\left(\beta_{1}\right) \leq W_{T}\left(\beta_{2}\right)$ iff $\hat{W}^{*}\left(\beta_{1}\right) \leq \hat{W}^{*}\left(\beta_{2}\right)$ for any $\beta_{1}, \beta_{2} \in \Theta$,

c. limiting distribution of $W_{T}\left(\beta_{0}\right)$ is same as that of $\hat{W}^{*}\left(\beta_{0}\right)$, and

d. $W_{T}(\beta)$ contours have data driven shape and they are centered around $\tilde{\beta}$. 
By incorporating the suggestion on transformation for the EL by Jing et al. (2017) to the AEL ratio statistic, we consider the following transformation:

$$
\hat{W}_{T}\left(\beta_{(1)}\right)=\hat{W}^{*}\left(\beta_{(1)}\right) \times \max \left\{1-\frac{\hat{W}^{*}\left(\beta_{(1)}\right)}{n+1}, 1-\gamma\right\},
$$

where $\gamma \in[0,1]$. As follows we check if the transformed adjusted empirical likelihood (TAEL) ratio satisfies the four conditions given above.

Condition a: We know that $\hat{W}^{*}\left(\beta_{(1)}\right)>0$. Therefore, if $\hat{W}^{*}\left(\beta_{(1)}\right)<\gamma(n+1)$, then $\max \left\{1-\frac{\hat{W}^{*}\left(\beta_{(1)}\right)}{n+1}, 1-\gamma\right\}=1-\frac{\hat{W}^{*}\left(\beta_{(1)}\right)}{n+1}$, and if $\hat{W}^{*}\left(\beta_{(1)}\right) \geq \gamma(n+1)$, then $\max \{1-$ $\left.\frac{\hat{W}^{*}\left(\beta_{(1)}\right)}{n+1}, 1-\gamma\right\}=1-\gamma$. Thus, $0<\max \left\{1-\frac{\hat{W}^{*}\left(\beta_{(1)}\right)}{n+1}, 1-\gamma\right\} \leq 1$. Furthermore,

$$
\hat{W}_{T}\left(\beta_{(1)}\right)=\left\{\begin{array}{lll}
\hat{W}^{*}\left(\beta_{(1)}\right) \times\left[1-\frac{\hat{W}^{*}\left(\beta_{(1)}\right)}{n+1}\right] & , \text { if } \quad \hat{W}^{*}\left(\beta_{(1)}\right)<\gamma(n+1) \\
\hat{W}^{*}\left(\beta_{(1)}\right)(1-\gamma) & \text {, if } \quad \hat{W}^{*}\left(\beta_{(1)}\right) \geq \gamma(n+1)
\end{array}\right.
$$

Condition b: We need to prove that the transformed function of AEL ratio, $\hat{W}_{t}\left(\beta_{(1)}\right)$ is a monotonically increasing function of $\hat{W}^{*}\left(\beta_{(1)}\right)$. We consider two cases.

i. When $\hat{W}^{*}\left(\beta_{(1)}\right) \in\left[0,(\gamma(n+1)]\right.$, then $\hat{W}_{T}\left(\beta_{(1)}\right)=\hat{W}^{*}\left(\beta_{(1)}\right) \times\left[1-\frac{\hat{W}^{*}\left(\beta_{(1)}\right)}{n+1}\right]$. So, $\hat{W}_{T}\left(\beta_{(1)}\right) \geq 0$ for all $\hat{W}^{*}\left(\beta_{(1)}\right)$, which implies that $\hat{W}_{T}\left(\beta_{(1)}\right)$ is monotonically increasing function of $\hat{W}^{*}\left(\beta_{(1)}\right)$.

ii. When $\hat{W}^{*}\left(\beta_{(1)}\right)>\gamma(n+1)$, then $\hat{W}_{T}\left(\beta_{(1)}\right)=\hat{W}^{*}\left(\beta_{(1)}\right) \times(1-\gamma)>0$, which implies that $\hat{W}_{T}\left(\beta_{(1)}\right)$ is monotonically increasing function.

Since $\hat{W}_{T}\left(\beta_{(1)}\right)$ is continuous at $\hat{W}^{*}\left(\beta_{(1)}\right)=\gamma(n+1)$, we can conclude that $\hat{W}_{T}\left(\beta_{(1)}\right)$ is a monotonically increasing function of $\hat{W}^{*}\left(\beta_{(1)}\right)$.

Condition c: Gamage et al. (2017) showed $\hat{W}^{*}\left(\beta_{(1)}\right)=o_{p}(1)$ in the equation (A.10). Thus with probability tending to unity, we have $\hat{W}^{*}\left(\beta_{(1)}\right) \leq(1-\gamma)(n+1)$. Combining them together we obtain,

$$
\hat{W}_{T}\left(\beta_{(1)}\right)=\hat{W}^{*}\left(\beta_{(1)}\right)+o_{p}(1) .
$$

Condition d: Let $\tau_{1} \in[0, \infty)$. Then the $\tau_{1}$-level contour of $\hat{W}_{T}\left(\beta_{(1)}\right)$ is $\left\{\beta_{(1)}: \hat{W}_{T}\left(\beta_{(1)}\right)=\right.$ $\left.\tau_{1}\right\}$. Now, if we consider the $\tau_{2}$-level contour of $\hat{W}^{*}\left(\beta_{(1)}\right),\left\{\beta_{(1)}: \hat{W}^{*}\left(\beta_{(1)}\right)=\tau_{2}\right\}$, to have the same shape as $\tau_{1}$-level contour, then we have $\left\{\beta_{(1)}: g_{T}\left(\hat{W}^{*}\left(\beta_{(1)}\right)\right)=\tau_{1}\right\}=\left\{\beta_{(1)}\right.$ : $\left.\hat{W}^{*}\left(\beta_{(1)}\right)=\tau_{2}\right\}$, where $g_{T}\left(\hat{W}^{*}\left(\beta_{(1)}\right)\right)=\hat{W}_{T}\left(\beta_{(1)}\right)$. Then, we get $\hat{W}^{*}\left(\beta_{(1)}\right)=g_{T}^{-1}\left(\tau_{1}\right)=$ $\tau_{2}$. This holds, since $\hat{W}_{T}\left(\beta_{(1)}\right)$ is a monotonically increasing function of $\hat{W}^{*}\left(\beta_{(1)}\right)$. This proves that the data driven shape is retained in the transformed statistic. Further, suppose at $\tilde{\beta}_{(1)}, \hat{W}^{*}\left(\beta_{(1)}\right)$ has a unique minimum, and also $\hat{W}_{T}\left(\beta_{(1)}\right)$ is monotonically increasing. Then, $\hat{W}_{T}\left(\tilde{\beta}_{(1)}\right)<\hat{W}_{T}\left(\beta_{(1)}\right)$ for $\beta_{(1)} \neq \tilde{\beta}_{(1)}$.

Since $\hat{W}_{T}\left(\beta_{(1)}\right)$ satistifies the conditions $(a)-(d)$ and $\hat{W}^{*}\left(\beta_{(1)}\right) \sim \chi_{p+q+1}^{2}$ (Gamage et al. 2017), $\hat{W}_{T}\left(\beta_{(1)}\right) \sim \chi_{p+q+1}^{2}$ as $n \rightarrow \infty$. We can similarly show that the transformed versions of the empirical likelihood with theoretical Bartlett correction (TB) and the empirical likelihood with estimated Bartlett correction (EB) satisfies the conditions (a)-(d), thus, both asymptotic distributions are $\chi_{p+q+1}^{2}$ as $n \rightarrow \infty$ for $\operatorname{ARFIMA}(\mathrm{p}, \mathrm{d}, \mathrm{q})$ model. We omit the details here. 


\section{Simulations}

In this section, we compare the coverage probabilities based on the transformed EL and AEL methods to the (untransformed) EL and AEL method as well as the EL with estimated Bartlett correction (EB) for models with different sample sizes and different distributions of error terms.

\subsection{Coverage probabilities}

\section{ARFIMA $(0, d, 0)$ model}

Monte Carlo simulations are conducted to compute the coverage probabilities to examine the efficiency and accuracy of the transformed EL and AEL methods for ARFIMA model. We compare the results to the coverage probabilities of the untransformed (or original) EL and AEL methods and EB. We consider the simplest model, $\operatorname{ARFIMA}(0, \mathrm{~d}, 0)$ given below:

$$
Z_{t}=(1-B)^{-d} a_{t}
$$

Simulations are carried out for different sample sizes, values of $d(d=(0.1,0.2,0.3,0.4))$ and three different distributions of the white noise term, namely, $N(0,1), t_{5}$, and $\chi_{5}^{2}$ distributions. In $t_{5}$ and $\chi_{5}^{2}$ distributions, the mean is subtracted from the white noise process in order to make the white noise process to have mean zero. Under each case, 1000 series of size $T=(50,70,100,200,1000)$ are drawn and the coverage probabilities are computed. We choose $a_{n}=\log (n) / 2$ as in the definition of $\psi_{n+1}$. The transformed empirical likelihood coverage probabilities and the transformed adjusted empirical likelihood coverage probabilities are compared to the coverage probabilities of the (untransformed) empirical likelihood and adjusted empirical likelihood and EB respectively. Table 1 shows the results for the simulations with a nominal level of $95 \%$. It can be seen that the coverage probabilities computed using the transformed EL and transformed AEL are higher than their untransformed counterparts. Comparing to EB, TAEL provides better coverage probabilities always while TEL performs better most times. Also when the sample size is small, the transformed AEL performs better and gives accurate results than the other methods.

ARFIMA $(0, d, 1)$ model

We consider ARFIMA $(0, \mathrm{~d}, 1)$ model:

$$
(1-B)^{d} Z_{t}=(1+\theta B) a_{t},
$$

where $a_{t}$ is the white noise process with mean zero and variance $\sigma^{2}$. The value of the model parameter, $\theta$ is set to be 0.5. We consider three different error distributions for the white noise process $a_{t}: \mathrm{N}(0,1), t_{5}$, and $\chi_{5}^{2}$. The last two distributions are centered around zero by subtracting their corresponding mean values. Four different $d$ values are considered: $d=(0.1,0.2,0.3,0.4)$. Table 2 shows that the coverage probability for models under different white noise distributions are different as it changes to adapt the differences in error distributions depicting the non-parametric property. Further, when the sample size is small, the transformed EL and AEL methods give better coverage probability as compared to the untransformed ones. Similar to Table 1, TAEL performs better in all scenarios while TEL performs better most times comparing to EB. 
Table 1: Coverage probabilities for the parameter of $\operatorname{ARFIMA}(0, \mathrm{~d}, 0)$ model

\begin{tabular}{|c|c|c|c|c|c|}
\hline$n$ & Method & $d=0.1$ & $d=0.2$ & $d=0.3$ & $d=0.4$ \\
\hline \multirow{6}{*}{$\mathrm{T}=50$} & \multicolumn{4}{|c|}{ Model: $a_{t} \sim N(0,1)$} & \\
\hline & EL & 0.670 & 0.668 & 0.675 & 0.69 \\
\hline & EL.T & 0.692 & 0.693 & 0.692 & 0.713 \\
\hline & AEL & 0.673 & 0.677 & 0.685 & 0.699 \\
\hline & AEL.T & 0.701 & 0.702 & 0.702 & 0.726 \\
\hline & $\mathrm{EB}$ & 0.680 & 0.683 & 0.690 & 0.705 \\
\hline \multirow{5}{*}{$\mathrm{T}=70$} & EL & 0.706 & 0.706 & 0.699 & 0.699 \\
\hline & EL.T & 0.072 & 0.716 & 0.710 & 0.716 \\
\hline & AEL & 0.712 & 0.713 & 0.705 & 0.712 \\
\hline & AEL.T & 0.727 & 0.724 & 0.718 & 0.730 \\
\hline & $\mathrm{EB}$ & 0.717 & 0.716 & 0.709 & 0.720 \\
\hline \multirow{5}{*}{$\mathrm{T}=100$} & EL & 0.749 & 0.746 & 0.741 & 0.747 \\
\hline & EL.T & 0.755 & 0.756 & 0.754 & 0.755 \\
\hline & AEL & 0.753 & 0.754 & 0.750 & 0.755 \\
\hline & AEL.T & 0.760 & 0.759 & 0.760 & 0.762 \\
\hline & $\mathrm{EB}$ & 0.753 & 0.755 & 0.754 & 0.758 \\
\hline \multirow{5}{*}{$\mathrm{T}=200$} & EL & 0.766 & 0.769 & 0.774 & 0.774 \\
\hline & EL.T & 0.769 & 0.773 & 0.778 & 0.776 \\
\hline & AEL & 0.768 & 0.772 & 0.780 & 0.777 \\
\hline & AEL.T & 0.774 & 0.780 & 0.783 & 0.779 \\
\hline & $\mathrm{EB}$ & 0.771 & 0.777 & 0.784 & 0.788 \\
\hline \multirow{5}{*}{$\mathrm{T}=1000$} & EL & 0.906 & 0.908 & 0.913 & 0.917 \\
\hline & EL.T & 0.907 & 0.909 & 0.916 & 0.919 \\
\hline & AEL & 0.908 & 0.913 & 0.921 & 0.930 \\
\hline & AEL.T & 0.908 & 0.914 & 0.921 & 0.931 \\
\hline & $\mathrm{EB}$ & 0.907 & 0.911 & 0.918 & 0.924 \\
\hline \multirow{6}{*}{$\mathrm{T}=50$} & \multicolumn{4}{|c|}{ Model: $a_{t} \sim t_{5}$} & \\
\hline & EL & 0.714 & 0.712 & 0.710 & 0.695 \\
\hline & EL.T & 0.732 & 0.722 & 0.722 & 0.707 \\
\hline & AEL & 0.720 & 0.715 & 0.714 & 0.700 \\
\hline & AEL.T & 0.738 & 0.733 & 0.730 & 0.720 \\
\hline & EB & 0.725 & 0.716 & 0.716 & 0.709 \\
\hline \multirow{5}{*}{$\mathrm{T}=70$} & EL & 0.719 & 0.727 & 0.732 & 0.738 \\
\hline & EL.T & 0.730 & 0.738 & 0.744 & 0.750 \\
\hline & AEL & 0.728 & 0.731 & 0.741 & 0.746 \\
\hline & AEL.T & 0.736 & 0.740 & 0.749 & 0.754 \\
\hline & EB & 0.729 & 0.733 & 0.742 & 0.750 \\
\hline \multirow{5}{*}{$\mathrm{T}=100$} & EL & 0.753 & 0.760 & 0.765 & 0.774 \\
\hline & EL.T & 0.761 & 0.767 & 0.775 & 0.779 \\
\hline & AEL & 0.757 & 0.763 & 0.771 & 0.778 \\
\hline & AEL.T & 0.768 & 0.771 & 0.784 & 0.787 \\
\hline & $\mathrm{EB}$ & 0.757 & 0.764 & 0.780 & 0.787 \\
\hline \multirow{4}{*}{$\mathrm{T}=200$} & EL & 0.788 & 0.787 & 0.788 & 0.776 \\
\hline & EL.T & 0.791 & 0.790 & 0.789 & 0.783 \\
\hline & AEL & 0.789 & 0.788 & 0.789 & 0.791 \\
\hline & AEL.T & 0.795 & 0.795 & 0.797 & 0.795 \\
\hline \multirow{6}{*}{$\mathrm{T}=1000$} & $\mathrm{~EB}$ & 0.790 & 0.792 & 0.794 & 0.796 \\
\hline & EL & 0.919 & 0.917 & 0.921 & 0.923 \\
\hline & EL.T & 0.921 & 0.918 & 0.922 & 0.923 \\
\hline & AEL & 0.921 & 0.920 & 0.931 & 0.936 \\
\hline & AEL.T & 0.921 & 0.921 & 0.932 & 0.936 \\
\hline & $\mathrm{EB}$ & 0.920 & 0.921 & 0.926 & 0.929 \\
\hline
\end{tabular}


Table 1 - continued from previous page

\begin{tabular}{|c|c|c|c|c|c|}
\hline$n$ & Method & $d=0.1$ & $d=0.2$ & $d=0.3$ & $d=0.4$ \\
\hline \multirow{6}{*}{$\mathrm{T}=50$} & & Model & $a_{t} \sim \chi_{5}^{2}$ & & \\
\hline & EL & 0.680 & 0.680 & 0.686 & 0.696 \\
\hline & EL.T & 0.703 & 0.705 & 0.701 & 0.713 \\
\hline & AEL & 0.690 & 0.691 & 0.694 & 0.703 \\
\hline & AEL.T & 0.714 & 0.717 & 0.709 & 0.721 \\
\hline & $\mathrm{EB}$ & 0.698 & 0.696 & 0.698 & 0.710 \\
\hline \multirow{5}{*}{$\mathrm{T}=70$} & EL & 0.722 & 0.726 & 0.723 & 0.722 \\
\hline & EL.T & 0.739 & 0.740 & 0.736 & 0.738 \\
\hline & AEL & 0.730 & 0.732 & 0.730 & 0.731 \\
\hline & AEL.T & 0.742 & 0.745 & 0.742 & 0.743 \\
\hline & EB & 0.732 & 0.734 & 0.733 & 0.736 \\
\hline \multirow{5}{*}{$\mathrm{T}=100$} & EL & 0.740 & 0.743 & 0.739 & 0.739 \\
\hline & EL.T & 0.751 & 0.753 & 0.749 & 0.751 \\
\hline & AEL & 0.746 & 0.751 & 0.745 & 0.750 \\
\hline & AEL.T & 0.752 & 0.758 & 0.761 & 0.756 \\
\hline & EB & 0.748 & 0.751 & 0.750 & 0.757 \\
\hline \multirow{4}{*}{$\mathrm{T}=200$} & EL & 0.782 & 0.790 & 0.791 & 0.789 \\
\hline & EL.T & 0.786 & 0.796 & 0.795 & 0.794 \\
\hline & AEL & 0.784 & 0.794 & 0.796 & 0.801 \\
\hline & AEL.T & 0.787 & 0.799 & 0.802 & 0.802 \\
\hline \multirow{6}{*}{$\mathrm{T}=1000$} & $\mathrm{~EB}$ & 0.785 & 0.795 & 0.799 & 0.808 \\
\hline & EL & 0.928 & 0.931 & 0.926 & 0.932 \\
\hline & EL.T & 0.929 & 0.933 & 0.929 & 0.933 \\
\hline & AEL & 0.929 & 0.934 & 0.94 & 0.94 \\
\hline & AEL.T & 0.932 & 0.935 & 0.941 & 0.942 \\
\hline & $\mathrm{EB}$ & 0.929 & 0.934 & 0.937 & 0.933 \\
\hline
\end{tabular}

$\mathrm{EL}=$ empirical likelihood;

$\mathrm{EB}=\mathrm{EL}$ with estimated Bartlett correction; AEL=Adjusted EL

'. $\mathrm{T}$ ' represents the transformed statistics 
Table 2: Coverage probabilities for the parameter of $\operatorname{ARFIMA}(0, \mathrm{~d}, 1)$ model

\begin{tabular}{|c|c|c|c|c|c|}
\hline$n$ & Method & $d=0.1$ & $d=0.2$ & $d=0.3$ & $d=0.4$ \\
\hline \multirow{6}{*}{$\mathrm{T}=50$} & & Model: a & $\sim N(0$ & & \\
\hline & EL & 0.312 & 0.418 & 0.321 & 0.311 \\
\hline & EL.T & 0.483 & 0.494 & 0.465 & 0.496 \\
\hline & AEL & 0.421 & 0.425 & 0.424 & 0.416 \\
\hline & AEL.T & 0.512 & 0.516 & 0.517 & 0.523 \\
\hline & $\mathrm{EB}$ & 0.410 & 0.411 & 0.413 & 0.417 \\
\hline \multirow{5}{*}{$\mathrm{T}=70$} & EL & 0.453 & 0.502 & 0.508 & 0.509 \\
\hline & EL.T & 0.543 & 0.543 & 0.543 & 0.542 \\
\hline & AEL & 0.522 & 0.523 & 0.524 & 0.525 \\
\hline & AEL.T & 0.557 & 0.554 & 0.553 & 0.558 \\
\hline & EB & 0.515 & 0.523 & 0.525 & 0.518 \\
\hline \multirow{5}{*}{$\mathrm{T}=100$} & EL & 0.546 & 0.622 & 0.624 & 0.623 \\
\hline & EL.T & 0.631 & 0.632 & 0.632 & 0.632 \\
\hline & AEL & 0.628 & 0.629 & 0.631 & 0.629 \\
\hline & AEL.T & 0.634 & 0.634 & 0.634 & 0.637 \\
\hline & $\mathrm{EB}$ & 0.601 & 0.628 & 0.629 & 0.628 \\
\hline \multirow{5}{*}{$\mathrm{T}=200$} & EL & 0.716 & 0.717 & 0.722 & 0.715 \\
\hline & EL.T & 0.742 & 0.738 & 0.739 & 0.737 \\
\hline & AEL & 0.736 & 0.734 & 0.732 & 0.73 \\
\hline & AEL.T & 0.752 & 0.752 & 0.748 & 0.741 \\
\hline & EB & 0.728 & 0.731 & 0.727 & 0.721 \\
\hline \multirow{5}{*}{$\mathrm{T}=1000$} & EL & 0.891 & 0.911 & 0.917 & 0.915 \\
\hline & EL.T & 0.925 & 0.921 & 0.921 & 0.921 \\
\hline & AEL & 0.909 & 0.914 & 0.933 & 0.925 \\
\hline & AEL.T & 0.938 & 0.937 & 0.937 & 0.932 \\
\hline & EB & 0.910 & 0.911 & 0.923 & 0.921 \\
\hline \multicolumn{6}{|c|}{ Model: $a_{t} \sim t_{5}$} \\
\hline \multirow{5}{*}{$\mathrm{T}=50$} & EL & 0.412 & 0.413 & 0.404 & 0.403 \\
\hline & EL.T & 0.581 & 0.582 & 0.581 & 0.573 \\
\hline & AEL & 0.599 & 0.599 & 0.597 & 0.596 \\
\hline & AEL.T & 0.606 & 0.607 & 0.607 & 0.605 \\
\hline & $\mathrm{EB}$ & 0.514 & 0.504 & 0.513 & 0.499 \\
\hline \multirow{5}{*}{$\mathrm{T}=70$} & EL & 0.612 & 0.609 & 0.604 & 0.603 \\
\hline & EL.T & 0.644 & 0.645 & 0.641 & 0.642 \\
\hline & AEL & 0.648 & 0.649 & 0.652 & 0.648 \\
\hline & AEL.T & 0.652 & 0.651 & 0.653 & 0.653 \\
\hline & $\mathrm{EB}$ & 0.629 & 0.633 & 0.626 & 0.622 \\
\hline \multirow{5}{*}{$\mathrm{T}=100$} & EL & 0.678 & 0.677 & 0.675 & 0.674 \\
\hline & EL.T & 0.684 & 0.686 & 0.678 & 0.676 \\
\hline & AEL & 0.686 & 0.683 & 0.682 & 0.682 \\
\hline & AEL.T & 0.686 & 0.686 & 0.685 & 0.686 \\
\hline & $\mathrm{EB}$ & 0.686 & 0.685 & 0.684 & 0.681 \\
\hline \multirow{4}{*}{$\mathrm{T}=200$} & EL & 0.778 & 0.777 & 0.775 & 0.772 \\
\hline & EL.T & 0.784 & 0.786 & 0.785 & 0.786 \\
\hline & AEL & 0.784 & 0.781 & 0.778 & 0.776 \\
\hline & AEL.T & 0.786 & 0.785 & 0.782 & 0.781 \\
\hline \multirow{6}{*}{$\mathrm{T}=1000$} & EB & 0.782 & 0.779 & 0.778 & 0.774 \\
\hline & EL & 0.896 & 0.893 & 0.897 & 0.892 \\
\hline & EL.T & 0.902 & 0.904 & 0.904 & 0.904 \\
\hline & AEL & 0.913 & 0.917 & 0.917 & 0.916 \\
\hline & AEL.T & 0.935 & 0.936 & 0.936 & 0.927 \\
\hline & EB & 0.909 & 0.904 & 0.906 & 0.906 \\
\hline
\end{tabular}


Table 2 - continued from previous page

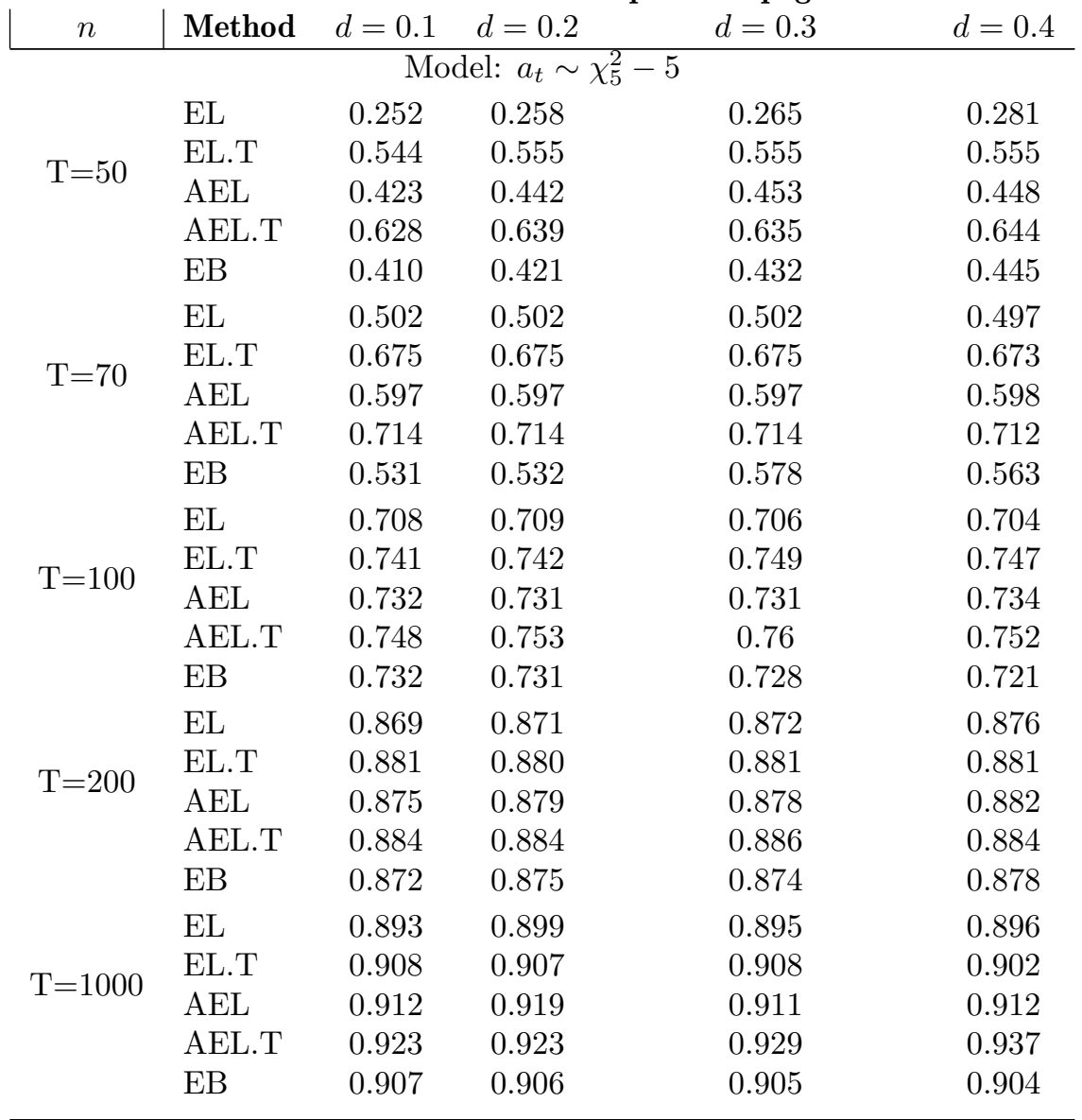

$\mathrm{EL}=$ empirical likelihood; EL.T=EL with the transformation;

$\mathrm{AEL}=$ Adjusted EL; AEL.T=EL with the transformation;

'.T' represents the transformed statistics 


\subsection{Power curves for ARFIMA $(1, \mathrm{~d}, 0)$ models}

For ARFIMA(1,d,0) model, we consider the behavior of power of the transformed empircal likelihood and transformed adjusted empirical likelihood under three different distributions for the white noise process $a_{t}: N(0,1), t_{5}$, and $\chi_{5}^{2}$. The latter two distributions are centered around zero. In these cases, we consider $\phi=0.2$ with four different values for $\mathrm{d}$ : $d=(0.1,0.2,0.3,0.4)$. We also consider four different sample sizes $(n=50,70,100,200)$ under each error distribution. Figure 1 below provides the power curves for ARFIMA(1,d,0) model at various sample sizes and orders of differencing $(d)$ for given error distributions. The black solid line is for the EL, red dotted line is for the EL with transformation, blue dotdash line is for the AEL and green dotdash line is for the AEL with transformation. It also indicates the patterns we observe from the Table $1 \&$ Table 2.

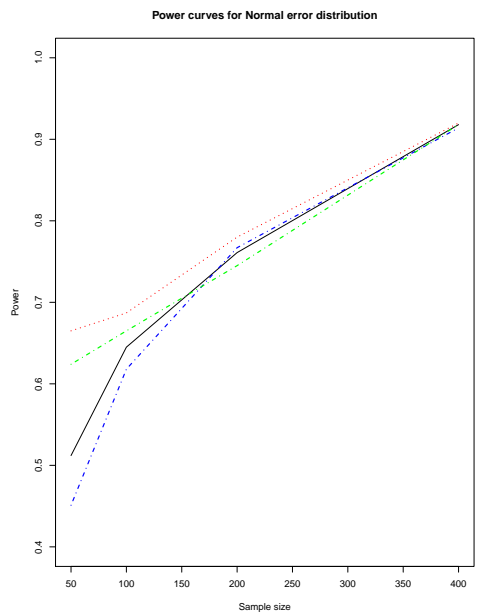

(a) $a_{t} \sim N(0,1)$

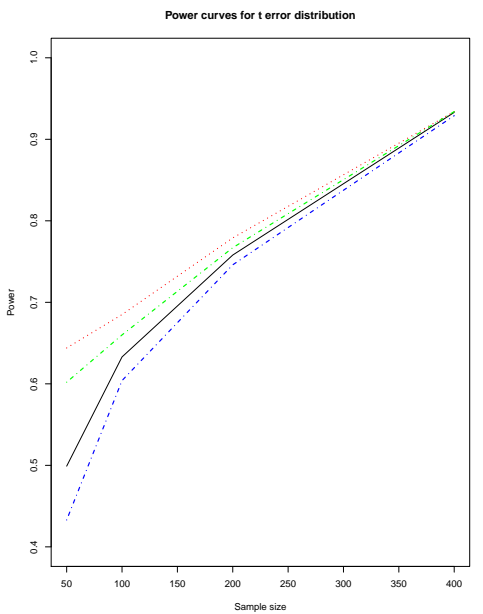

(b) $a_{t} \sim t_{5}$

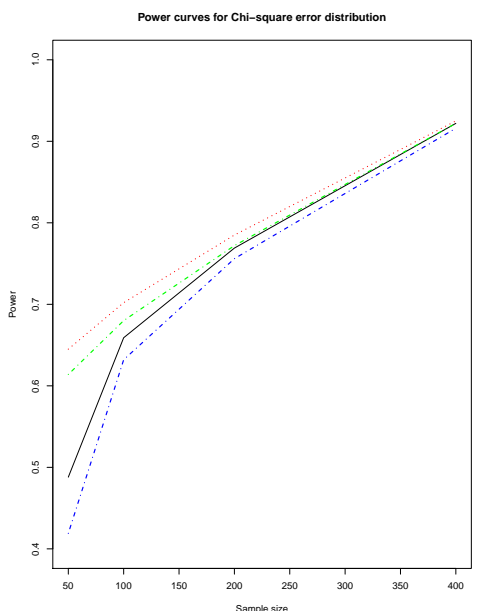

(c) $a_{t} \sim \chi_{5}^{2}$

Figure 1: Power curves for $\operatorname{ARFIMA}(1, \mathrm{~d}, 0)$ models

\section{Discussion}

In this paper, we propose a transformed adjusted empirical likelihood (TAEL) to extend the method by Gamage et al. (2017) for long-memory time series models, specifically ARFIMA models. Such a method combines the advantage of the adjusted empirical likelihood (AEL) by Chen et al. (2008) on ensuring the existence of the solutions in optimizing the profile empirical likelihood ratio, and the advantage of the transformed empirical likelihood (JEL) by Jing et al. (2017) on improving coverage probabilities while maintaining the same asymptotic properties and simple form. The asymptotic null distribution of the TAEL statistic for long-memory time series models has been established as a standard chi-square distribution. Simulations have been conducted for different ARFIMA models with various sample sizes and error distributions to compare the performance between the proposed TAEL and the other existing methods. Results indicate that the TAEL provides better coverage probabilities especially for small sample sizes.

\section{Acknowledgements}

The authors would like to thank the anonymous referee for his/her constructive comments and suggestions which helped to improve this manuscript significantly.

\section{References}

Chen J, Variyath AM, Abraham B (2008). "Adjusted Empirical Likelihood and Its Properties." Journal of Computational and Graphical Statistics, 17(2), 426-443. URL https://doi.org/10.1198/ $106186008 \times 321068$. 
Gamage RDP, Ning W, Gupta AK (2017). "Adjusted Empirical Likelihood for Long-memory Timeseries Models." Journal of Statistical Theory and Practice, 11(1), 220-233. URL https://link. springer.com/article/10.1080/15598608.2016.1271373.

Hurvich CM, Beltrao KI (1993). "Asymptotics for the Low-frequency Ordinates of the Periodogram of a Long-memory Time Series." Journal of Time Series Analysis, 14(5), 455-472. URL https: //doi.org/10.1111/j.1467-9892.1993.tb00157.x.

Jing BY, Tsao M, Zhou W (2017). "Transforming the Empirical Likelihood towards Better Accuracy." Canadian Journal of Statistics, 45(3), 340-352. URL https://doi.org/10.1002/cjs.11328.

Kitamura Y (1997). "Empirical Likelihood Methods with Weakly Dependent Processes." The Annals of Statistics, 25(5), 2084-2102. URL https://projecteuclid.org/download/pdf_1/euclid.aos/ 1069362388.

Monti AC (1997). "Empirical Likelihood Confidence Regions in Time Series Models." Biometrika, 84(2), 395-405. URL https://doi.org/10.1093/biomet/84.2.395.

Mykland PA (1995). "Dual Likelihood." The Annals of Statistics, pp. 396-421. URL https://www. jstor.org/stable/2242343.

Nordman DJ, Lahiri SN (2006). "A Frequency Domain Empirical Likelihood for Short-and Long-range Dependence." The Annals of Statistics, 34(6), 3019-3050. URL https://projecteuclid.org/ download/pdfview_1/euclid.aos/1179935073.

Owen AB (1988). "Empirical Likelihood Ratio Confidence Intervals for a Single Functional." Biometrika, 75(2), 237-249. URL https://doi.org/10.1093/biomet/75.2.237.

Owen AB (1990). "Empirical Likelihood Ratio Confidence Regions." The Annals of Statistics, 18(1), 90-120. URL https://projecteuclid.org/download/pdf_1/euclid.aos/1176347494.

Owen AB (1991). "Empirical Likelihood for Linear Models." The Annals of Statistics, pp. 1725-1747. URL https://www.jstor.org/stable/2241901.

Owen AB (2001). Empirical Likelihood. Chapman and Hall/CRC. URL https://www.crcpress .com/ Empirical-Likelihood/Owen/p/book/9781584880714.

Tsao M (2013). "Extending the Empirical Likelihood by Domain Expansion." Canadian Journal of Statistics, 41(2), 257-274. URL https://doi.org/10.1002/cjs.11175.

Yau CY (2012). "Empirical Likelihood in Long-memory Time Series Models." Journal of Time Series Analysis, 33(2), 269-275. URL https://doi.org/10.1111/j.1467-9892.2011.00756.x, .

\begin{abstract}
Affiliation:
Ramadha D. Piyadi Gamage

Department of Mathematics

Western Washington University

Bellingham, WA 98225, USA

E-mail: piyadir@wwu .edu
\end{abstract}

Wei Ning

Department of Mathematics and Statistics

Bowling Green State University

Bowling Green, OH 43403, USA

E-mail: wning@bgsu.edu

\begin{tabular}{lr}
\hline Austrian Journal of Statistics & http://www.ajs.or.at/ \\
published by the Austrian Society of Statistics & http://www.osg.or.at/ \\
Volume 49 & Submitted: 2019-06-06 \\
August 2020 & Accepted: $2019-11-28$ \\
\hline
\end{tabular}

